\title{
Modelo de jardín vertical con vegetación endémica para espacios públicos exteriores en El Grillo, Jalisco
}

Vertical garden model with endemis vegetation for external public spaces en El Grillo, Jalisco

Recibido: febrero 10 de 2020 | Revisado: enero 17 de 2021 | Aceptado: febrero 19 de 2021

\author{
Alethia Nefertari Chávez Gutiérrez ${ }^{\mathrm{I}}$ \\ Francisco José Martín del Campo SARAY ${ }^{\mathrm{I}}$ \\ Gonzalo Bojórquez Morales ${ }^{2}$
}

\begin{abstract}
Resumen
La preocupación ambiental en escala global ha impulsado la tendencia del "movimiento verde" para actuar en contra de factores como el calentamiento global, la contaminación del agua y del aire y pérdida del hábitat (Buraglia et al., 2011). Para este estudio, se hace un enfoque específico en los sistemas de jardinería vegetal aplicado al espacio público exterior como alternativa para mejoramiento y aumento de áreas verdes. Para ello, se propuso el diseño de un modelo de jardín vertical con plantas endémicas dentro de la ciudad de El Grullo, Jalisco, México, que pueda adaptarse a condiciones meteorológicas y espaciales del lugar. El objetivo de esta investigación fue proponer un modelo de jardín vertical con vegetación endémica para espacios públicos exteriores. La metodología que se utilizó fue observacional, descriptiva y transversal, para la factibilidad del estudio, previa bibliografía especializada, se tomó como referencia el análisis de la vegetación y mobiliario público que circunda las áreas de estudio. Se señala lo relevante de la investigación, donde refiere que los jardines verticales con vegetación endémica propician una mejor imagen visual de espacios verdes para la comunidad de El Grullo, donde se favorece la socialización, bienestar y convivencia de los habitantes a estos espacios.
\end{abstract}

Palabras clave: jardín vertical, vegetación endémica, espacios públicos exteriores

\begin{abstract}
Environmental concern on a global scale has fueled the trend of the "green movement" to act against factors such as global warming, air and water pollution, and loss of habitat (Buraglia et al., 2011). For this study, a specific focus is made on the vegetable gardening systems applied to the public outdoor space, as an alternative for improvement
\end{abstract}

(C) Los autores. Este artículo es publicado por la Revista Campus de la Facultad de Ingeniería y Arquitectura de la Universidad de San Martín de Porres. Este artículo se distribuye en los términos de la Licencia Creative Commons Atribución No-comercial - Compartir-Igual 4.0 Internacional (https://creativecommons.org/licenses/ CC-BY), que permite el uso no comercial, distribución y reproducción en cualquier medio siempre que la obra original sea debidamente citada. Para uso comercial contactar a: revistacampus@usmp.pe. 
and increase of green areas. For this, the design of a vertical garden model with endemic plants within the city of El Grullo, Jalisco, Mexico, which can adapt to the meteorological and spatial conditions of the place, was proposed. The objective of this research was to propose a vertical garden model with endemic vegetation for outdoor public spaces. The methodology that was used was observational, descriptive and cross-sectional, for the feasibility of the study, after specialized bibliography, the analysis of the vegetation and public furniture that surrounds the study areas was taken as a reference. The relevant aspect of the research is pointed out, where it refers that the vertical gardens with endemic vegetation promote a better visual image of green spaces for the community of El Grullo, where the socialization, well-being and coexistence of the inhabitants are favored in these spaces.

Key words: vertical garden, endemic vegetation, outdoor public spaces

\section{Introducción}

La aglomeración de asentamientos humanos y la industrialización rápida en conjunto son factores que generan un efecto negativo sobre el entorno natural, pues estos son capaces de acelerar el proceso de urbanización de una ciudad. Pero si no se tiene un plan de desarrollo urbano adecuado para controlar dicho crecimiento, la movilidad toma una posición relevante, siendo capaz de desplazar los espacios públicos y/o de convivencia, lo que trae como consecuencia una urbe carente de áreas verdes. En ese contexto, la planificación de espacios verdes para las actividades recreativas y el descanso, es un elemento integrante de un problema general: la protección del equilibrio de la naturaleza. La salud física y mental del hombre, depende en medida de la protección del patrimonio natural, lo que exige a su vez una planificación cuidadosa, pues la función principal de estas áreas verdes, es lograr escenarios adecuados para actividades recreativas y sociales, que son de gran importancia para el ambiente físico, biodiversidad y calidad del aire (Mejía y Gómez, 2015).

El Programa de las Naciones Unidas por el Medio Ambiente (PNUMA), expone que las áreas verdes urbanas son componentes primordiales para mejorar el bienestar de la población urbana, especialmente, en ciudades grandes. Sin embargo, son escasas en las ciudades de América Latina, producto de la historia de urbanización precaria y explosiva de la segunda mitad del siglo XX (Reyes y Figueroa, 2010). Adicionalmente, como medida de regularización ante la reducción de vegetación en los núcleos urbanos, la Organización Mundial de la Salud (OMS) estableció un estándar de nueve $\mathrm{m}^{2}$ por habitante como medida recomendable mínima, dada la importancia de las áreas verdes para la calidad de vida de la población urbana (Ídem, p. 91).

En 2012, se realizó un análisis con base en un satélite Spot 2009 perteneciente 
al Instituto Nacional de Estadística y Geografía (INEGI) para verificar si el municipio de Guadalajara, Jalisco, México cumplía con el parámetro de la OMS, y se llegó a la conclusión que el 34 $\%$ de la población vive en zonas críticas o con carencias severas de vegetación, pues tienen menos de cuatro $\mathrm{m}^{2}$ de áreas verdes por habitante (Instituto de Información Territorial del Estado de Jalisco, 2012). Otro ejemplo de la falta de atención y enfoque en el desarrollo de las áreas verdes, espacios recreativos y parques urbanos, es la localidad de El Grullo, Jalisco, México, pues debido a la falta de mantenimiento y atención por parte de las autoridades, estos espacios no logran ser óptimos para generar una calidad en el ambiente del sistema urbano, lo que puede ocasionar islas de calor, poca asistencia a los espacios públicos exteriores por parte de los habitantes, imagen urbana deficiente e incremento potencial de la delincuencia y vandalismo.

La planificación cuidadosa y previsión de las necesidades de la población son claves para asegurar que una ciudad tenga recursos naturales sanos para el presente y el futuro. Es por ello, que los planificadores urbanos necesitan la participación comunitaria en el proceso de toma de decisiones ya que los espacios verdes son para el gozo y beneficio de todos los ciudadanos. Esto significa, involucrar al público en aspectos que varían desde la selección de sitios y diseño de los espacios verdes, hasta establecer por rango de prioridades los beneficios ambientales deseados y desarrollar estrategias factibles para mantenerlos (Sorensen et al., 1997). Como parte de las estrategias, la restauración ecológica funge como la serie de acciones encaminadas a reducir, mitigar y revertir el deterioro de la naturaleza. Tiene la finalidad de integrar en un área determinada, elementos biológicos adecuados, los cuales pueden ser nativos o exóticos a un paisaje local, sin pretender regresar al sistema de vida tradicional (Mendoza y Cano, 2009). Además, cabe resaltar la importancia del paisaje dentro de una localidad, pues se concibe como un recurso de valor estético que combina elementos físicos, bioecológicos y humanos; y representa un conjunto de interrelaciones derivadas de las interacciones entre geomorfología, clima, vegetación, fauna, agua y modificaciones antrópicas (Gómez et al., 2013). Como estrategia para impulsar el mantenimiento de las áreas verdes, el desarrollo urbano sostenible, integra la jardinería vertical con objetivos de propósito ornamental, ya que son capaces de aportar naturación de la ciudad, aumento de la biodiversidad, producción de alimentos, control del flujo de agua de lluvia, aislamiento acústico y térmico, además de propiciar el ahorro energético (Fernández et al., 2008).

La creación de un índice de habitabilidad térmica en espacios de convivencia públicos exteriores en clima cálido semiseco, se llevó a cabo en El Grullo, Jalisco, México, donde se analizó la vegetación de las áreas de intervención (Jardín municipal y Alameda municipal) y su contexto urbano, donde se tiene un área estimada de $678 \mathrm{~m}^{2}$ de vegetación promedio para los dos espacios públicos, además, se contempló el promedio de áreas verticales y horizontales de elementos circundantes que pueden influenciar la zona de estudio (Martín del Campo et al., 2020).

En comparativa, la población de El Grullo, Jalisco, México, con un total 
de 22,499 personas de acuerdo al censo de población del Instituto Nacional de Estadística y Geografía (2010) y al estándar de vegetación establecido por la OMS, la ciudad debería de contar con 20 hectáreas de vegetación aproximadamente (Instituto de Información Estadística y Geográfica, 2018). Una táctica ante la falta evidente de vegetación dentro de la zona urbana, se propone un modelo de jardín vertical para los espacios públicos exteriores, como un complemento de las áreas verdes dentro de la urbe, debido a que existen pocos espacios que lo implementan y al uso de vegetación que no pertenece a la zona de estudio que favorezca la convivencia en estos lugares. Para ello, se enfoca en tres factores fundamentales para su desarrollo: el ambiental, el tecnológico y el social. En el factor ambiental, se seleccionó la vegetación que promueva el confort y que a su vez sea ornamental para generar un impacto positivo en su imagen urbana; en el factor tecnológico, se buscó reducir el mantenimiento en costo monetario, mediante la aplicación de un sistema de riego autónomo con base a la recolección de agua, y por último, el factor social, que de manera asociada con los anteriores, atendió los problemas de habitabilidad para satisfacer sus necesidades de recreación y convivencia.

Desde otra perspectiva como parte del estado del Arte, en el continente europeo, en el ańo 2017, en la ciudad de Sevilla, España, se elaboró un estudio del diseño, construcción y análisis de funcionamiento inicial de un sistema de acuaponía que combina un estanque ornamental con un jardín vertical exterior. Para iniciar el proyecto, se elaboró un croquis para planificar las tareas a realizar, que tiene en cuenta la disponibilidad de los materiales (Fernández y Pérez, 2007). En 2016 en Madrid, España, se realizó un estudio comparativo de sustratos de sistemas de naturación urbana, debido al encarecimiento, a la baja disponibilidad de sustratos existentes y a la creciente demanda de los más específicos. Por ello, como objetivo del estudio, fue caracterizar, comparar y evaluar el comportamiento y la evolución de sustratos elaborados a partir de materiales sostenibles, potenciales para su utilización como medio de crecimiento vegetal (López, 2016).

Como parte de estrategias que promuevan la eficiencia energética, se realizó un trabajo de investigación, en donde se compararon sistemas eficientes y benéficos para mejorar el confort de los usuarios mediantela aplicación desistemas de jardinería vertical, concentrándose en los efectos sobre el edificio y el medio ambiente (Olivieri, 2013). Por otro lado, en el continente asiático, en enero del 2015, se elaboró un documento sobre una breve discusión sobre los sistemas de vegetación vertical en Hong Kong como parte de una iniciativa, para la respuesta de la contaminación del aire severa y el efecto de isla de calor generado por los asentamientos humanos. Discute los beneficios ambientales, económicos $\mathrm{y}$ sociales que traen los sistemas de jardinería vertical, sus aplicaciones y las especies más utilizadas en la ciudad, con el propósito de mejorar la calidad del entorno construido (Dahanayake y Lun, 2015).

Con el propósito de promover los principios sostenibles en los espacios públicos, en 2014 se publicó el artículo “'Sistemas de jardinería vertical como arte público? Posibilidades y desafíos en 
el contexto urbano de Malasia", el cual reúne nueve proyectos existentes en espacios públicos de la urbe, en donde se exploraron dimensiones de la vegetación vertical, los tipos de arte público y como se adaptan al entorno, que demuestran varios tipos, sistemas y métodos de vegetación vertical (Abu Bakar et al., 2014). Otra investigación, se propuso el uso de sistemas de vegetación vertical, como una estrategia para reducir la transmisión de calor, dividiéndolo en dos categorías principales: fachadas verdes y paredes vivas (Safikhani et al., 2014). Con respecto a la aplicación de sistemas de jardinería vertical en el continente americano, Ochoa (2019), en la ciudad de la Cuenca, Ecuador, elaboró un sistema de diseño de jardines con la finalidad de llevar la vegetación a los espacios interiores y exteriores de exposición, sean públicos o privados. Su objetivo fue implementar un sistema constructivo, dinámico, versátil y funcional, para el soporte de las plantas naturales, donde incluyó un análisis sobre las mismas para su mejor adaptación.

A su vez, en Córdoba, Argentina, se desarrolló una investigación sobre la eficiencia del uso de muros verdes para disminuir los efectos negativos de la pérdida de áreas verdes en las ciudades con el uso de materiales reciclados, ya que, los muros verdes pueden contribuir a la gestión de aguas pluviales, calidad del aire, reducción de la temperatura y mitigación del efecto isla de calor (Rivas, 2019). Por otro lado, en la ciudad de Punta Arenas, Chile, en 2015, el Instituto de Investigaciones Agropecuarias (INIA), publicó un texto informativo sobre la construcción de muros verdes, o sistemas de jardinería vegetal, mediante sistemas modulares metálicos con relleno de musgo Sphagnummagellanicu, una opción de sustrato viable, debido a que lo consideraban liviano, poroso y con buena retención de agua, además de poseer un buen anclaje para las raíces de las plantas y poca compactación (Domínguez, 2015).

Asimismo, en 2015 en Medellín, Colombia, se desarrolló un estudio exploratorio acerca de jardines ornamentales urbanos contemporáneos, para la incorporación de vegetación en los espacios públicos, como parte de su proceso de renovación y expansión.

Se analizó la vegetación ornamental no arbórea contenida en 143 jardines públicos de la zona suroriental de Medellín, y mediante muestreo estadístico, se determinó la composición florística y las características paisajísticas de la vegetación; y se obtuvo un total de 198 especies, de las cuales 158 resultaron exóticas (Vélez y Herrera, 2015).

Como fusión de los jardines verticales y la agricultura urbana, en 2012 nacieron los Módulos para Huertas Urbanas Verticales (MHUV) como un sistema alternativo que beneficia a la producción de alimentos libres de químicos, no transgénicos, regados con agua potable y 100\% orgánicos (Navas y Peña, 2012). Desde otra perspectiva, dentro de los pocos estudios realizados en México, en Acapulco, Guerrero, se realizó una investigación denominada "Captura de carbono por una fachada vegetada", que tuvo por objeto determinar la cantidad de carbono y dióxido de carbono, capturado por las especies Pentalinonluteum y Clitoriaternatea, utilizadas en el modelo construido (Carbajal et al., 2017). El método utilizado para determinar el carbono capturado por $\mathrm{m}^{2}$, se basó en la producción de biomasa, durante un 
periodo de seis meses para la especie Clitoriaternatea, mientras que para la especie Pentalinonluteum, fue de un año y seis meses. Para la equivalencia del dióxido de carbono, se tuvo en consideración, que cada mol de $\mathrm{CO}_{2}$ contiene 12 gramos de carbono, lo que indica que, por cada gramo de carbono asimilado, se retiran de la atmósfera 3.66 gr de $\mathrm{CO}_{2}$ (Ídem, p. 56).

Desde el enfoque psicológico, se elaboró una investigación acerca de la percepción sobre un sistema de fachada vegetada, en estudiantes y docentes de educación superior en Acapulco, Guerrero, México. La construcción del modelo consistió en un sistema tipo enrejado, con un recipiente metálico para el sustrato, con una altura y profundidad de $30 \mathrm{~cm}$ y un lardo de dos metros. Se cubrió por dentro y por fuera con pintura anticorrosiva y se colocaron dos capas de plástico en el interior para protegerlo de la humedad. Para la guía y crecimiento de la vegetación se utilizó una reja prefabricada de $2 \times 2,5 \mathrm{~m}$ hecha de alambre galvanizado, con acabado de poliéster para evitar la corrosión y el calentamiento; se ancló por medio de una solera en sus extremos y se separó $5 \mathrm{~cm}$ de la superficie del muro para facilitar la ventilación y evitar la saturación de humedad. Las plantas que se utilizaron fueron enredaderas nativas de las regiones tropicales de América Pentalinonluteum y Clitoriaternatea, la mezcla del sustrato fue compuesta con piedra tepetate triturada a $1 \mathrm{~cm}$ de granulometría aproximadamente, fibra de coco, humus de lombriz y abono de hoja. La superficie total vegetada fue de $5 \mathrm{~m} 2$ (Carbajal et al., 2016).

En cuanto a los resultados de las entrevistas, acerca de los beneficios que provee un muro verde, se obtuvo un 60.6
$\%$ para temperatura interior, un $18.5 \%$ para limpieza del aire, un $9.7 \%$ para la apariencia del edificio, un $7.3 \%$ para el paisaje urbano y para otros un $4,0 \%$. Ante las bondades del muro vegetativo, los participantes del sondeo consideraron que los inmuebles en los cuales se debería instalar esta tecnología son las casas $(39,5 \%)$ y escuelas $(28,2 \%)$ (Ibidem, p. $269-270)$.

Ahora, el concepto de "Jardín vertical" como tal, fue patentado en 1988, por el botánico francés Patrick Blanc, al presentar su estructura elaborada con un marco de metal, una placa de PVC y dos capas de fieltro poliamida, modelo que revolucionó la aplicación de la vegetación en la edificación, gracias a su sistema modular que era capaz de adaptarse en cualquier área y a su sistema de tubería adherida al panel de PVC que proporcionaba agua para su mantenimiento (De la Cruz, 2014). Los jardines verticales son diseñados con la intención de aportar beneficios diversos además del ambiental y ornamental ya que se vuelven un elemento multifuncional para el espacio, es capaz de transformarse en estrategia sustentable y en mobiliario urbano, además, propicia el ahorro energético, es un sustituto de áreas verdes y aporta beneficios al confort urbano.

Los sistemas de jardinería vertical han reunido significados y nomenclaturas desde su aparición, la vegetación vertical es aquella que está integrada en formas construidas, considera balcones, jardines, terrazas y techos verdes. Estos autores clasificaron los sistemas en dos categorías de acuerdo a su aplicación. Los sistemas de soporte, los cuales son para guiar las plantas en sentido vertical sobre la superficie, mientras que los 
sistemas portadores, están diseñados para contener el sustrato y los medios para plantar la vegetación de manera vertical (Badrulzaman et al., 2013).

Por tanto, se podría decir que los sistemas de jardinería vertical se crearon con la finalidad de integrar un espacio natural dentro de las ciudades de manera eficiente, por lo que estos se desarrollan sobre una superficie no convencional en la que se pueda adaptar al poco espacio existente, debido al crecimiento desordenado de la población. Esto les permite integrarse a una edificación o un muro, para aprovechar las virtudes de la vegetación y el aporte estético. Si bien, los jardines verticales son sistemas con gran versatilidad, consideran el desarrollo y evolución de la tecnología en muros verdes, por ello, es importante identificar y clasificar los sistemas diferentes, de acuerdo a su lugar de aplicación, a sus componentes y las técnicas de construcción.

El jardín se reconoce como un elemento de gran importancia en los asentamientos humanos, ya que constituye una representación estética y cultural que conlleva valores de tipo ambiental. Diseñar el paisaje con la biodiversidad incorporada dentro de la ciudad, se considera un espacio neutro desde el punto de vista ecológico, debido a que la urbanización conlleva cierta pérdida de especies y hábitats (Vélez y Herrera, 2015).

Por ello, el paisajismo representa la conexión y versatilidad socio-espacial, que permite analizar en escalas múltiples los patrones y procesos que le son propios, con la capacidad de abordar áreas rurales, ecosistemas silvestres, redes e infraestructuras, así como planificar extensiones vacías o urbanizadas (Moreno, 2013). Ahora bien, la naturación urbana es definida como la transformación de los edificios y espacios urbanos de condiciones ambientales uniformes, uniéndolos mediante corredores verdes, que facilitan la circulación atmosférica y mejora el microclima de la ciudad (Fernández et al., 2015). Se menciona, que para finalizar con este capítulo se formuló la pregunta de investigación ¿Cuál estrategia resulta eficiente para la preferencia de los habitantes en el espacio público exterior de El Grullo, Jalisco, México? De la que se derivó la hipótesis; el jardín vertical con vegetación endémica propicia una visita y permanencia mayor de los habitantes en el espacio público exterior de El Grullo, Jalisco, México. De acuerdo a lo anterior, el objetivo de la investigación fue proponer un modelo de jardín vertical con vegetación endémica para espacios públicos exteriores de $\mathrm{El}$ Grullo, Jalisco.

\section{Método}

Para la factibilidad del estudio se tomó como referencia el análisis de la vegetación y mobiliario público que circunda el Jardín Municipal "Ramón Corona” y la Alameda Municipal. Este tipo de metodología fue observacional, descriptiva y transversal. Para el registro de datos se auxilió mediante una cámara fotográfica personal y cédula de información de datos diseñada para la captura de estas variables.

Con el fin de realizar la investigación, se propuso que el objeto de estudio fueran espacios públicos exteriores de la comunidad de El Grullo, Jalisco, México, que se localiza en la región Sierra 
de Amula del Estado de Jalisco, México, con una latitud de $19^{\circ} 48^{\prime} 22.80^{\prime \prime} \mathrm{N}$ $104^{\circ} 13^{\prime} 09.29^{\prime \prime} \mathrm{O}$, colinda al norte con el municipio de Unión de Tula, Ejutla y El Limón, Jalisco; al este con los municipios de El Limón y Tuxcacuesco, Jalisco; al sur con los municipios de Tuxcacuesco y Autlán de Navarro, Jalisco; al oeste con el municipio de Autlán de Navarro y Unión de Tula, Jalisco (Secretaría del Medio Ambiente y Desarrollo Territorial, 2013). Figura 1.

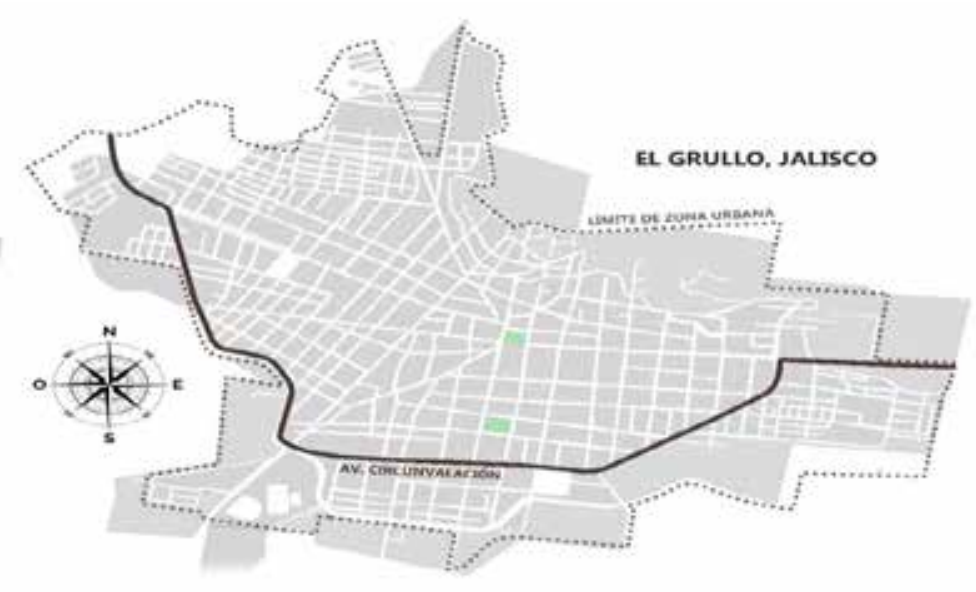

Figura 1. Vista área de El Grullo, Jalisco

Este municipio se caracteriza por tener un tipo de clima cálido semiseco, aunque algunos meses del año las condiciones meteorológicas lo cambian a subhúmedo influido por el temporal de lluvias, la temperatura máxima promedio es de $35^{\circ} \mathrm{C}$, la mínima promedio de $11^{\circ} \mathrm{C}$ y la temperatura media anual es de $23.9^{\circ} \mathrm{C}$ con una precipitación media anual de $900 \mathrm{~mm}$, una altitud de 876 m.s.n.m. y humedad relativa promedio del $35 \%$ (Instituto Nacional de Estadística, Geografía e Informática, 1996).

Se tomó para el muestreo dos lugares representativos de El Grullo, Jalisco, México, (Jardín Municipal "Ramón Corona” y Alameda Municipal). Estos lugares son espacios públicos exteriores de El Grullo, Jalisco, México. Para el diseño de la cédula de información de vegetación y mobiliario se propuso manejarlo por apartados para facilitar la captura y análisis de los datos obtenidos, con base en ello, se propuso un modelo de jardín vertical que atienda las necesidades funcionales del espacio en estudio. Con el uso de la cámara fotográfica se obtuvieron larguillos de imágenes de las áreas de espacios públicos por puntos cardinales.

Para la realización del muestreo, se recolectaron 72 cédulas de información, en las cuales se recogieron datos acerca del tipo de mobiliario y la especie a la que pertenece la vegetación, por lo cual se caracterizaron de acuerdo a sus dimensiones, forma, textura, copa de árbol, sombra de árbol, nivel de deterioro y material, según correspondiera. Por otra parte, para la realización del análisis en el lugar de estudio, el sitio se dividió en sectores con base en el punto de observación (norte, sur, oriente y poniente).

Con respecto a la clasificación de acuerdo al tipo de vegetación, se contabilizó por especie existente en cada sector, catalogándolos en cubre suelo, 
arbustos y árboles. Para la clasificación por tipo de especie, se investigó el nombre común y el número de vegetación que existe en cada punto, registrándolas por unidad, por lo que para el cubre suelo (pasto San Agustín) no aplica, ya que no se puede registrar por unidad, debido a que su unidad de medida es diferente. Cabe aclarar que las especies variaron de acuerdo al sector que se analizó.

\section{Análisis de las áreas de estudio}

Derivado de ello, en el sector norte del Jardín municipal, se obtuvieron 32 árboles, mientras que en la Alameda fueron 41 árboles y dos arbustos; en el sector sur, el Jardín municipal tuvo 29 árboles y la Alameda municipal 45 árboles; en el sector oriente, el Jardín municipal dio un total de 100 árboles, mientras que en la Alameda hubo 53 árboles y dos arbustos, y al final, en el sector poniente, el Jardín municipal tuvo 28 árboles y en la Alameda municipal fueron 45. En la mayoría de los casos, la Alameda municipal presentó vegetación mayor, y aunque en el sector oriente el Jardín municipal presenta un total de 100 árboles, 48 son palmas, donde se analizó, que es un tipo de vegetación no adecuada por las condiciones meteorológicas para El Grullo, Jalisco, México.

Desde otra perspectiva, en el análisis de dimensiones de la vegetación, se establecieron cinco rangos de altura [1] 0.01 a 0.30 , [2] 0.31 a 1.00, [3] 1.01 a 2.00, [4] 2.01 a 3.00 y de [5] 3.01 o más metros, cuantificándolos por unidad con base en el total obtenido de la vegetación por especie, para lo cual se incluyeron las características de cubre suelo. En el sector norte y sur del Jardín municipal, predominó la altura de 1.01 a 2.00 metros, mientras que en el sector oriente y poniente, fue de 3.01 o más metros. En la Alameda municipal en el sector norte, sur y oriente, la dimensión principal fue de 2.01 a 3.00 metros, mientras que en el sector oriente, la altura sobresaliente fue de 3.01 o más metros.

En el análisis de la forma, se examinó la copa del árbol o arbusto, mientras que para el cubre suelo se tomó como ejemplo la superficie en la que se aplicó. De acuerdo a la forma se clasificó en plana, cónica, cilíndrica, redonda y trapezoidal. En el Jardín municipal, la forma plana fue constante en el sector norte, sur y oriente, mientras que en el sector poniente, la forma trapezoidal dominó. En contraste, en la Alameda la forma plana tuvo un número mayor de apariciones en los cuatro sectores.

En la variable de textura de la vegetación, se estudió el tronco del árbol y los arbustos, mientras que para el cubre suelo se examinó el tipo de superficie. Dicha característica se dividió en tres tipos de texturas: lisa, intermedia y rugosa. Como resultado, la textura intermedia sobresalió en los cuatro sectores de las dos áreas de estudio.

En el estudio de las dimensiones de copa de árbol, se establecieron tres rangos: [1] de 0.50 a 1.50 , [2] 1.51 a 2.50 y [3] 2.51 o más metros, aquí no se tomó en cuenta el cubre suelo, debido al tipo de variable. La dimensión de copa de árbol más frecuente en el sector norte, sur y oriente en el Jardín municipal, fue de 1.51 a 2.50 metros, mientras que en el poniente fue de 2.51 o más metros. Por otra parte, en la Alameda municipal, en el sector norte, sur y poniente, la dimensión de copa de árbol más común, fue de 2.51 
o más metros, de igual modo en el sector oriente, fue de 1.51 a 2.50 metros.

Con respecto a las dimensiones de la sombra de un árbol, se establecieron tres promedios de las dimensiones: [1] 0.50 a 1.50 , [2] 1.51 a 2.50 y de [3] 2.51 o más metros. En el Jardín municipal, la dimensión de sombra de 2.51 o más metros, predominó en el sector norte, mientras que en el sector oriente la dimensión principal fue de 1.51 a 2.50 metros, y al final, en el sector oriente hubo una paridad entre 0.50 a 1.50 y 2.51 o más metros. Por otra parte, en la Alameda municipal, la sombra predominante fue de 2.52 o más metros y para el sector oriente fue de 1.51 a 2.50 metros.

El último aspecto a evaluar en la vegetación fue su estado de deterioro, dividiéndolo en cuatro categorías como pésimo, regular, bueno y excelente con la finalidad de examinar si la implementación y el mantenimiento de las áreas verdes eran satisfactorios en ambos espacios públicos exteriores. Como resultado del análisis del nivel de deterioro en la vegetación, en el Jardín municipal en el sector norte, la vegetación fue de consideraciones buenas, en el sector sur se clasificó como excelente, mientras que en el sector oriente y poniente fue regular. En la Alameda municipal, en el sector norte, sur, y oriente, la vegetación fue regular, mientras que en el poniente fue en su mayoría buena. También como parte del análisis de estudio, se valoró el mobiliario y sus características. En primera instancia, el mobiliario se catalogó en cinco tipos: bancas, jardineras, fuentes, aparatos de acondicionamiento físico y aparatos de acondicionamiento recreativo.
En el sector norte del Jardín municipal, se tuvo en total 12 bancas, cuatro jardineras y una fuente, en el sector sur dio como resultado 19 bancas, ocho jardineras y una fuente; en el sector oriente hubo ocho bancas y tres jardineras, y en el sector poniente se tuvieron 23 bancas y dos jardineras. Así mismo, en la Alameda municipal en el sector norte hubo 18 bancas, en el sector sur 22, en el sector oriente 23 y en el sector poniente dio un total de 20 bancas y una fuente.

Para continuar, al igual que con la vegetación, se examinaron las dimensiones del mobiliario, clasificándolo en cinco rangos, para lo que se tomó en cuenta el largo o el diámetro. Como resultado para el Jardín municipal, en el sector norte, el resultado mayor fue de 12 bancas con una dimensión de 1.01 a $2.00 \mathrm{~m}$, para el sector sur, oriente y poniente, la dimensión predominante fue de 2.01 a $3.00 \mathrm{~m}$ con un total de 18,8 y 23 bancas respectivamente. En comparación, para la Alameda municipal, en sector norte y sur, sobresalió la dimensión de 3.01 o más metros, 16 y 22 bancas respectivamente, mientras que para el sector oriente y poniente, la dimensión constante fue de 2.01 a 3.00 metros, con 15 y 14 bancas.

Para el análisis de la forma de mobiliario, se establecieron seis tipos, denominados como: plana, cónica, cilíndrica, trapezoidal, cuadrada y rectangular. Los datos resultantes para el Jardín municipal, en el sector norte, sur y oriente, proyectaron que la forma de frecuencia mayor fue la rectangular, con un total de 12, 19 y ocho unidades de mobiliario, de acuerdo al orden en el que se mencionó; sin embargo, para el sector oriente, la forma que predominó fue la cuadrada, con 23 unidades de 
mobiliario. Mientras tanto, para la Alameda municipal, en el sector norte, sur, oriente y poniente, la forma que destacó fue la plana, con 20, 22, 23 y 19 unidades de mobiliario respectivamente.

En la variable siguiente se estudió la textura del mobiliario, para lo cual se examinó el tipo de material de composición y se clasificó en tres tipos de textura: lisa, intermedia y rugosa. Como resultado para el Jardín municipal, en los cuatro sectores se estableció como principal la textura lisa, ya que la mayoría del mobiliario era de metal, en contraste con la Alameda municipal, sobresalió la textura intermedia, ya que el material constructivo para mobiliario en su mayoría fue de concreto.

El siguiente punto compara el tipo de material del mobiliario, para lo que se consideró el concreto, metal, plástico, madera, cristal y polímero. Como se mencionó en el apartado anterior, en el Jardín municipal, el mobiliario metálico destacó en los cuatro sectores con 12, 19, 8 y 23 unidades de mobiliario. Así mismo, en la Alameda municipal, el material de frecuencia mayor fue el concreto, con un total de 19, 22, 23 y 20 bancas, en los cuatro sectores.

Para finalizar con el análisis de los datos, el último punto a evaluar del mobiliario, fue su nivel de deterioro, que al igual que la vegetación se clasifico en pésimo, regular, bueno y excelente. En el Jardín municipal, en el sector norte, sur y poniente, el mobiliario tuvo calidad regular, que en comparación con el sector oriente, fue bueno. A su vez, en la Alameda municipal, los resultados proyectaron que, para los cuatro sectores, en su mayoría se clasificó al mobiliario como regular. Dicho lo anterior, el análisis de este aspecto del mobiliario, tuvo la finalidad de determinar la propuesta del jardín vertical, era necesario incluir mobiliario, por lo que, al clasificarse como un punto intermedio, la propuesta fue a elección.

\section{Resultados y Discusión}

Con base en el análisis de las variables y los resultados expuestos se determinaron tres propuestas para cubrir las necesidades de los espacios públicos exteriores, cada una adaptándose a diferentes situaciones. La primera propuesta del modelo de jardín vertical, se elaboró en base a los resultados de la Alameda municipal, donde existe un número elevado de vegetación en la mayoría de los sectores, por lo que el jardín vertical se concentra en generar un ambiente más cómodo en el aspecto visual y de confort, mediante el paso del aire a través del modelo.

Señalado lo anterior, se propuso un sistema de jardín vertical móvil, con la finalidad de que el usuario pueda manipularlo para generar un entorno más cómodo. Por ello, el sistema consiste en una estructura metálica elaborada con perfiles tubulares de $2.0 \times 2.0$ pulgadas, con un calibre de $12 \mathrm{~mm}$, al cual se anclan cinco jardineras de fibra de vidrio con dimensiones de $1.20 \times 0.30 \times 0.30$ $\mathrm{cm}$ en la base y para que el modelo pueda desplazarse, se ańaden cuatro ruedas movibles de poliuretano con un diámetro de $10 \mathrm{~cm}$.

El sustrato a usar para los tres modelos, se propuso con base en la documentación expuesta, por lo que se planteó un sustrato con $50 \%$ de fibra de coco y $20 \%$ de restos vegetales compostados para generar 
mayor humedad, aireación y aprovechar sus propiedades drenantes, debido a que el sustrato orgánico tiene una mejor retención de agua López (2016), mientras que el otro $30 \%$ restante, se complementó con tierra y humus de lombriz, como protector de la irradiación solar y para mitigar el efecto invernadero (Ramírez y Davis, 2014). La planta a utilizar para la primera propuesta fue la especie Monstera deliciosa, perteneciente a la familia Araceae, conocida comúnmente como Costilla de Adán. Esta planta se desarrolla mejor en climas húmedos o semihúmedos. Es preferible que permanezca bajo condiciones de sombra y tolera de manera moderada la sequía (Guillén, 2018). Figura 2.

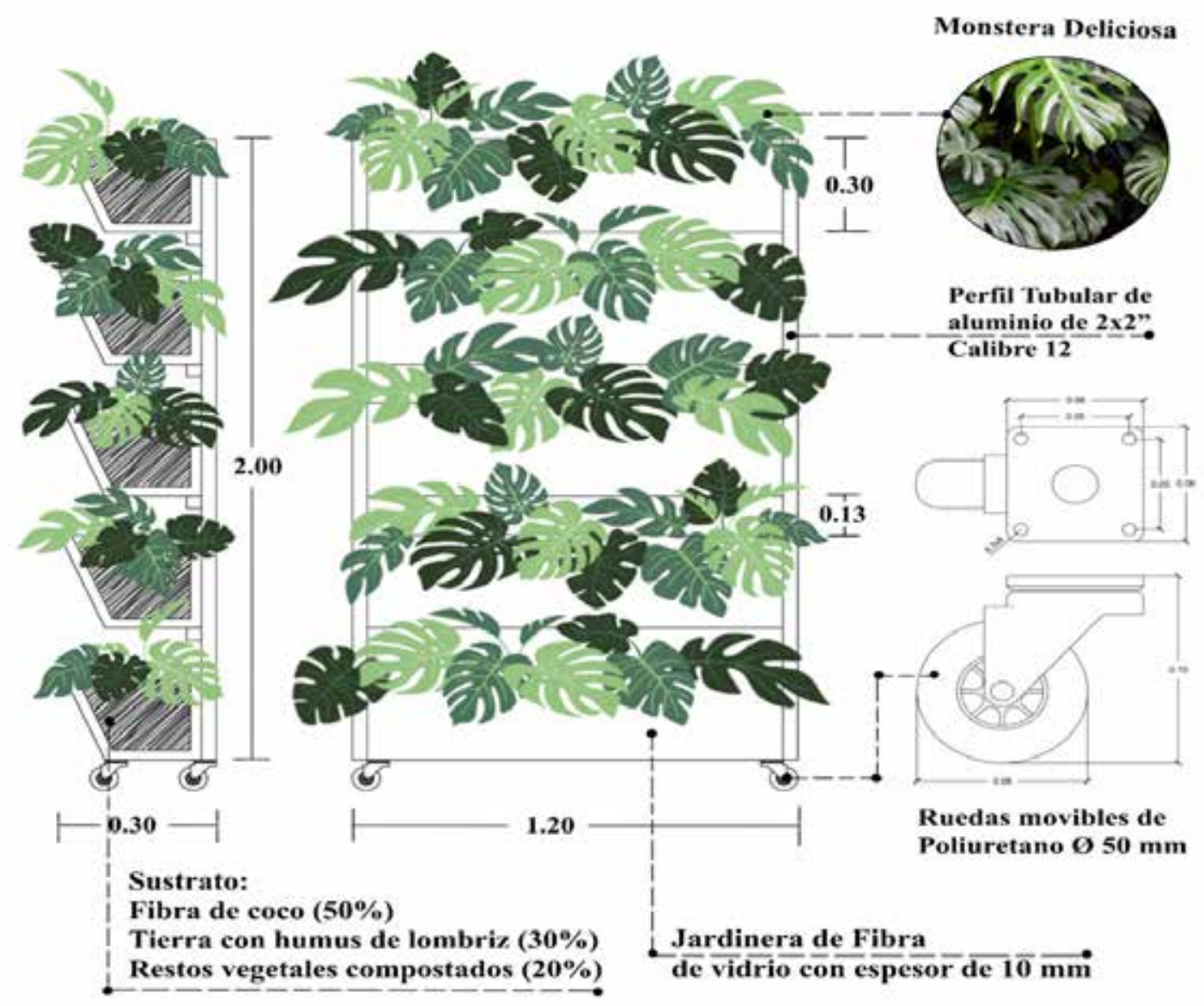

Figura 2. Propuesta 1 - Jardín vertical móvil

En la segunda propuesta del modelo de jardín vertical, se utilizó el mismo concepto del anterior, a diferencia de que la vegetación no se implementa de la misma manera. Este sistema, consiste en una estructura de perfiles tubulares metálicos de 2.0 x 2.0 pulgadas con calibre de $12 \mathrm{~mm}$, con cables de acero galvanizado anclados con argollas tornillo de $1 / 4$ de pulgada. En la parte baja, se implementa una jardinera de fibra de vidrio con el mismo tipo de sustrato, en la cual se coloca una planta perenne trepadora, denominada por su nombre científico como Ficus Pumila, mejor conocida como Higuera trepadora, que forma parte de la familia de las Moráceas. Pertenece a clima cálido y requiere una cantidad moderada de agua para su crecimiento (Starr et al., 2003). Figura 3. 


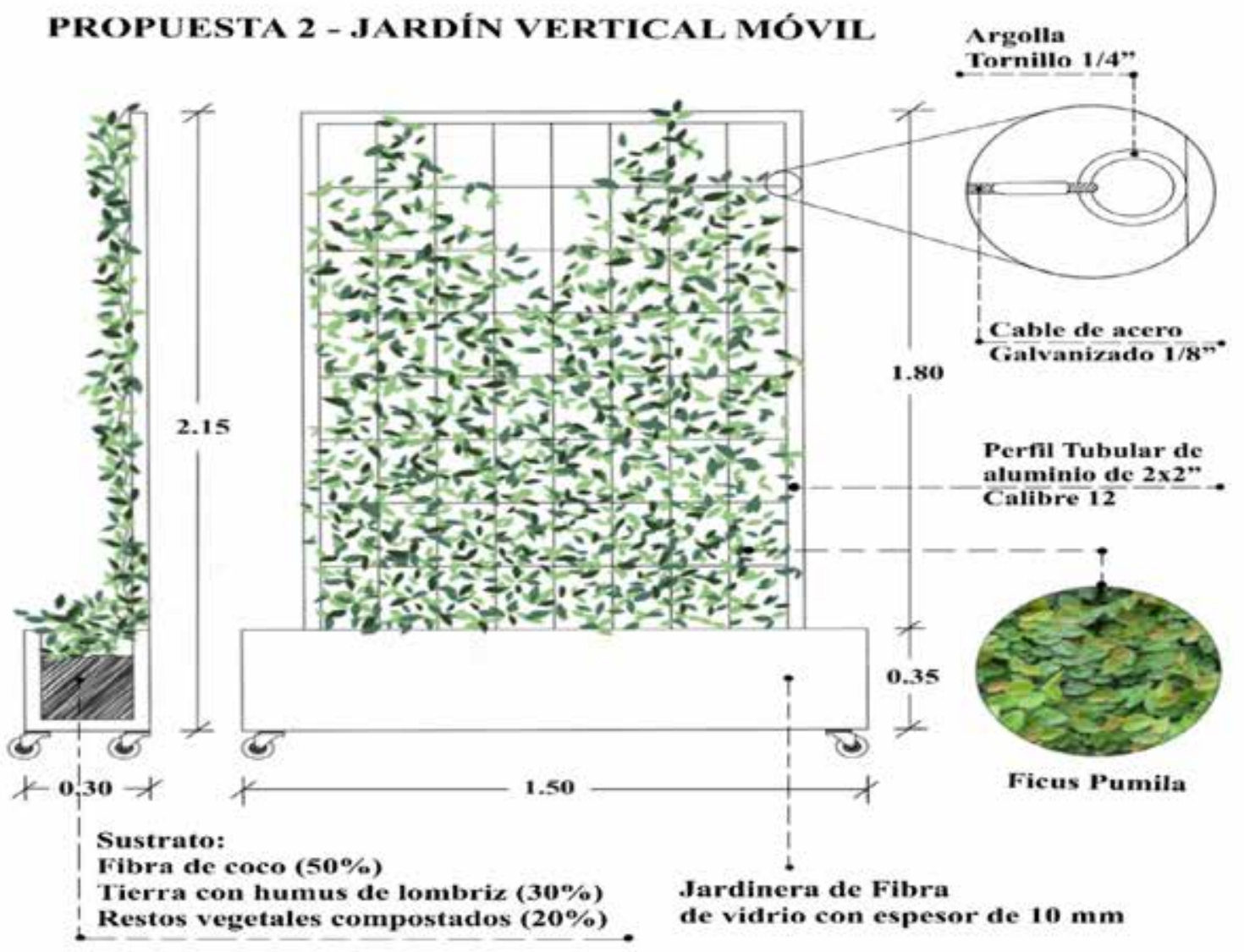

Figura 3. Propuesta 2 - Jardín vertical móvil

En la tercera propuesta, se planteó un jardín vertical integrado al mobiliario, esto debido a los resultados obtenidos en el análisis, ya que en su mayoría el mobiliario resultó con un nivel de deterioro regular, y se consideró que este aspecto afectaba el confort del usuario al asistir al espacio. El modelo consistió en un muro de concreto, sobre el cual se coloca ladrillo cerámico Berdea que cuenta con un compartimiento para colocar el sustrato. Se complementa con una estructura metálica anclada al ladrillo que soporta un pergolado de perfiles tubular de aluminio de $2.0 \mathrm{x}$
2.0 pulgadas con calibre de $12 \mathrm{~mm}$, cubierto de pintura vinílica con textura de madera, mientras que en la parte baja, se propone un mobiliario formado con perfiles tubulares y una colchoneta de goma espuma, resistente a la intemperie. La vegetación a utilizar fue el helecho filicopsida o helecho macho, familia de las Polypodiaceae. Esta planta es capaz de adaptarse a cualquier tipo de clima y requiere una cantidad moderada de agua, y tiene la ventaja de que puede adecuarse a cualquier espacio (Barzola y Renzo, 2018). Figura 4. 
PROPUESTA 3 - JARDIN VERTICAL CON MOBILIARIO
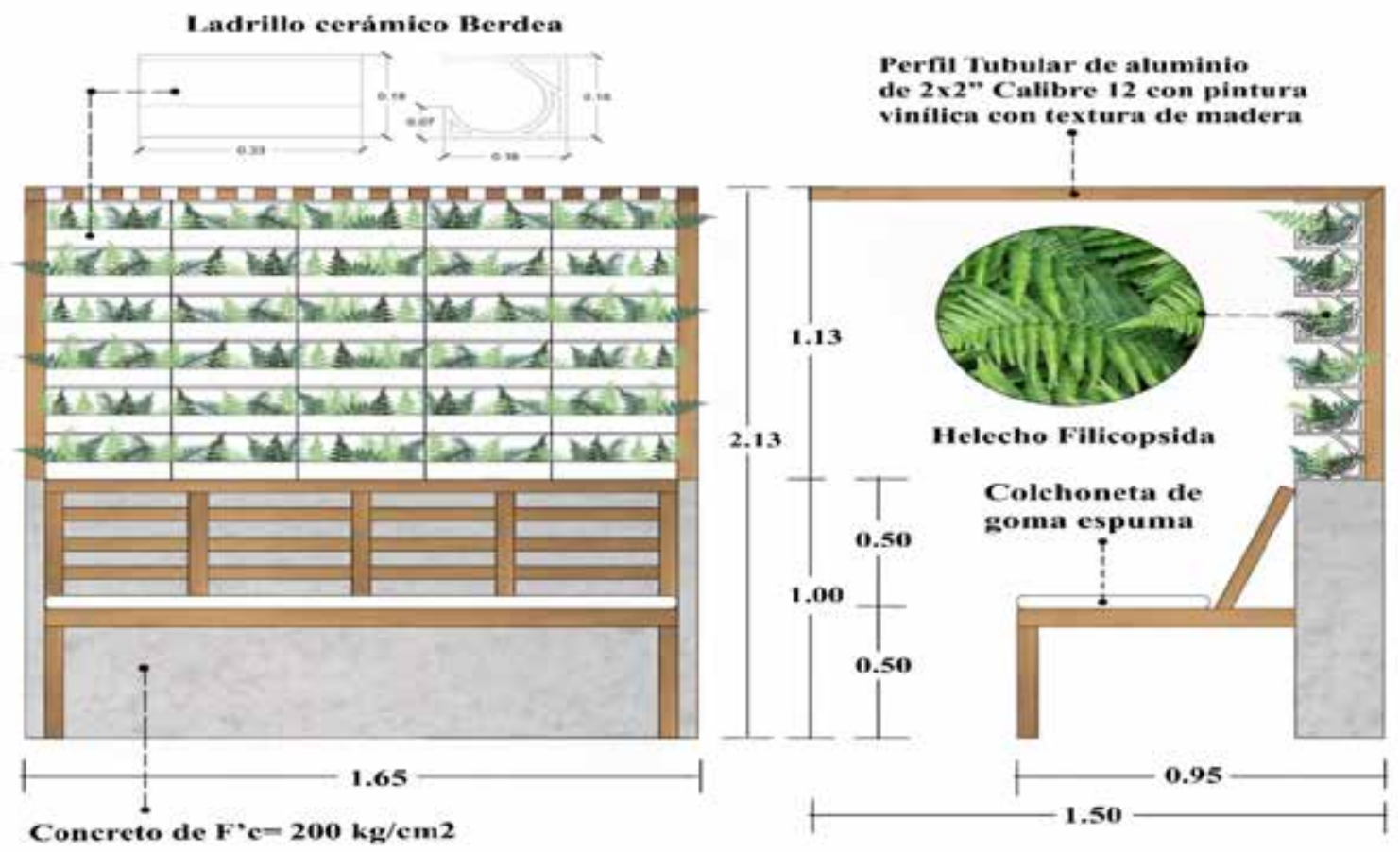

Figura 4. Propuesta 3-Jardín vertical con mobiliario

\section{Conclusiones}

Se señala lo relevante de la investigación donde refiere que los jardines verticales con vegetación endémica propician una mejor imagen visual de espacios verdes para la comunidad de El Grullo, Jalisco, donde se favorece la socialización, bienestar y convivencia de los habitantes a estos espacios. El objetivo fundamental del estudio fue proponer un modelo de jardín vertical con vegetación endémica para espacios públicos exteriores de El Grullo, Jalisco, México.

Con relación a la preferencia de los habitantes que asisten al Jardín municipal, se encontró que su percepción sobre este espacio posee una vegetación deficiente, debido a que, presenta un solo tipo de cubre suelo y ausencia de arbustos, lo que genera que el ambiente térmico no resulte satisfactorio para sus usuarios.
En lo que respecta a la preferencia de habitantes que asisten a la Alameda municipal señalaron que, aunque presenta mayor vegetación y de características mejores que el Jardín municipal, el mobiliario tiene un deterioro mayor y esto a su vez, repercute en la comodidad de la estancia de los usuarios y asimismo, degrada la imagen visual del entorno urbano. Con base en el análisis de estudio y los resultados obtenidos, se validó la hipótesis que se presentó en la investigación y todos los objetivos planteados fueron cumplidos satisfactoriamente. Sobre los resultados del tipo de vegetación existente en la Alameda municipal que posee un número mayor de árboles que el Jardín municipal, se detectó que estas especies no brindan el sombreado requerido por la copa de sus árboles, para un parámetro de confort térmico de sus habitantes, que, de acuerdo a recomendaciones de 
bibliografía especializada, los árboles de hoja perenne son más adecuados para este tipo de clima. Sobre la forma de árbol que se presentó con frecuencia mayor en ambas áreas de estudio fue la plana, seguida por la trapezoidal.

Esta situación explica que, de acuerdo a estas características, no favorece el sombreado requerido en zonas de mobiliario en periodo de verano; por consiguiente, se apreció un nicho de oportunidad en el mejoramiento visual y espacial de espacios públicos exteriores con la propuesta del modelo de jardín vertical con vegetación endémica que circunda las áreas de caminamientos, de distribución, de convivencia y mobiliario público. La propuesta número 1 es un modelo de jardín vertical basado en el sistema de contenedores, por lo cual, se consideraron cinco contenedores de fibra de vidrio anclados a una estructura de aluminio con ruedas de poliuretano, para su desplazamiento a necesidad de las condiciones meteorológicas y espaciales que se requieran.

Sobre la propuesta número 2 , el sistema de modelo de jardín vertical, se basó en un sistema de soporte de enrejado metálico con una base de fibra de vidrio, sobre la cual, lleva el sustrato para propiciar el crecimiento de enredaderas vegetativas; $y$ de esta manera, mitigue el impacto ambiental. En lo que respecta a la propuesta número 3 de jardín vertical, se determinó integrarlo al mobiliario de descanso con una tendencia innovadora de material constructivo que resulte confortable y de forma ergonómica; por tal motivo, se diseńó una estructura de perfil tubular de aluminio de $2.0 \mathrm{x}$ 2.0 pulgadas con calibre de $12 \mathrm{~mm}$, acompañado de un colchón de goma espuma resistente a la intemperie. Se sugiere que este tipo de estudios tenga un seguimiento puntual en otro tipo de clima y un análisis a profundidad con los usuarios de espacios públicos exteriores, para obtener a detalle su percepción contextual sobre la temática abordada. Además, este prototipo de estudios ayuda a que los organismos municipales, tomen en cuenta al habitante y su contexto urbano para renovar y remodelar estas áreas o en su caso diseñar espacios nuevos donde se consideren todos los aspectos señalados, con la finalidad de mejorar la asistencia y permanencia por parte de los individuos a dichas áreas.

\section{Agradecimientos}

Se agradece a los directivos del Instituto Tecnológico José Mario Molina Pasquel y Henríquez Campus El Grullo, por la buena disposición en apoyar este trabajo de investigación y la difusión de investigaciones similares, que permiten una formación integral de sus estudiantes y egresados. 


\section{Referencias}

Abu Bakar, et al. (2014). Vertical Greenery System as Public Art? Possibilities and challenges in Malaysian urban context. Kuala Lumpur, Malaysia: Department of Landscape Architecture, Kulliyyah of Architecture and Environmental Design.

Badrulzaman, J., et al. (2013). Impact of Vertical Greenery System on Internal Building. Skudai, Malaysia: Elsevier Ltd.

Barzola, H., y Renzo, R. (2018). Fitoextraccion del Cadmio en los Relaves Oxidados mediante el uso del Helecho (Microsorum scolopendria) Lima 2018. Lima, Perú: Universidad César Vallejo.

Buraglia, M., et al. (2011). Jardínes verticales y sistemas botánicos en entornos urbanos. MasD Revista Digital de Diseño(Edición 9), 51-63.

Carbajal Avila , J., et al. (2016). percepción por un modelo de fachada vegetada. Acapulco, México: Centro de Investigación Científica y Tecnológica de Guerrero A.C. (CICTEG).

Carbajal Avila, J., et al. (2017). Captura de carbono por una fachada vegetada. Acta Universitaria: Multidisciplinary Scientific Journal, 55-61.

Dahanayake, K. C., yLun Cho, C. (2015). $A$ brief discussion on current vertical greenery systems in Hong Kong: the way forward. Hong Kong, China:
Departament of Architecture and Civil Engeneering, City University of Hong Kong.

De la Cruz García, R. (2014). Jardinería ornamental y hortícola. Espańa: Ministerio de Educación, cultura y deporte.

Domínguez, E. (2015). Jardines Verticales usando Musgo Sphagnum. Punta Arenas: Institución de Investigaciones Agropecuarias (INIA).

FérnandezCañero, R., yPérezUrrestarazu, L. (2017). Diseño, construcción y análisis de funcionamiento inicial de un sistema de acuaponía que combina con un estanque ornamental con un jardin vertical exterior. Sevilla, España: Universidad de Sevilla, Escuela Técnica Superior de Ingenieria Agronómica.

Fernández Cañero, R., et al. (2015). Naturación Urbana y Jardinería vertical. PARJAP - Revista de Asociación Española de parques $y$ jardínes públicos, 7.

Fernandez Cañero, R., et al. (2008). Ajardinamiento de fachadas $y$ jardines verticales: otras formas de jardinería aplicadas a un desarrollo urbano sostenible. Sevilla, España: Departamento de Ingeniería Aeroespacial y Mecánica de Fluidos. EUITA, Universidad de Sevilla.

Gomez Merino, F. C., et al. (2013). Paisaje y turismo rural en México: fortalezas y desafíos para su 
potenciación. Revista Mexicana de Ciencias Agricolas, 1028.

Guillén Carvajal, M. J. (2018). Determinacion del estado de desarrollo óptimo para la cosecha de Monstera Deliciosa, Philodendron $s p$. cv. Xanadú y Philodendron sp. cv. Xantal. Costa Rica: Facultad de Ciencias Agroalimentarias.

Instituto de Información Estadística y Geográfica. (2018). El Grullo, Diagnóstico de Municipio. Guadalajara, Jalisco: Gobierno de Jalisco.

Instituto de Información Territorial del Estado de Jalisco. (2012). Cantidad de áreas verdes de Guadalajara y Tlaquepaque por abajo de lo recomendado. Guadalajara, Jalisco: Gobierno de Jalisco.

Instituto Nacional de Estadística, Geografía e Informática. (1996). Cuaderno Estadístico Municipal. Guadalajara: Gobierno del Estado de Jalisco.

Köhler, M. (2008). Green facades: a view back and some visions. Neubrandenburg, Germany: Springer Science + Business Media.

López Rodriguez, G. L. (2016). Estudio comparativo de sustratos de cultivo sostenibles en sistemas de naturación urbana: Jardinería vertical y Cubierta vegetal. Madrid, España: Universidad Politécnica de Madrid.

Martín del Campo Saray, F. J., et al. (2020). Tesis doctoral "Índice de habitabilidad térmica en espacios de convivencia públicos exteriores en clima cálido semiseco". Universidad de Colima. Coquimatlán, Colima: Programa Interistitucional de Doctorado de Arquitectura.

Mejía Salazar, G., y Gómez, R. (2015). Un análisis a la superficie de área verde por habitante en la ciudad de Tepic, Nayarit, México. Invertigación Joven: Revista Electrónica, 1.

Mendoza Hernández, P. E., y Cano Santana, Z. (2009). Elementos para la restauración ecológica de pedregales: la rehabilitación de áreas verdes de la Facultad de Ciencias en Ciudad Universitaria. Ciudad de México: Departamento de Ecología y Recursos Naturales, Facultad de Ciencias de la Universidad Autónoma de México.

Moreno Flores, O. (2013). Paisaje, riesgo y resilencia. La arquitectura del paisaje en la modelacion sustentable del territorio. Forum de Sostenibilidad, 17-30.

Navas Navarro, F. H., y Peña Torres, L. M. (2012). Los diseños verticales y la agricultura unidos para la producción de alimentos en los Módulos para Huertas Urbanas Verticales. Zona centro de Bógota, Colombia : Escuela de Ciencias Agrícolas, Pecuarias y de Medio Ambiente ECAPMA.

Ochoa Farjado, E. A. (2019). Sistema de diseño de jardines itinerantes. Cuenca, Ecuador. : Universidad de Azuay, Facultad de Arquitectura, Diseño y Arte. 
Olivieri, F. (2013). Caracterización experimental y modelo predictivo del comportamiento térmico de una fachada vegetal. Madrid, España: Universidad Politécnica de Madrid.

Ramírez C. , F., y Marks Davis, M. (3 de noviembre de 2014). Muro Orgánico Urbano Silvestre Sostenible. Revista PUCE(98), 134.

Reyes Päcke, S., y Figueroa Aldunce, I. M. (2010). Distribución, superficie y accesibilidad de las áreas verdes en Santiago de Chile. EURE, 89-110.

Rivas Sánchez, Y. A. (2019). Eficiencia del uso de muros verdes para disminuir los efectos negativos de la perdidad de áreas verdes en las ciudades con el uso de materiales reciclados. Córdoda, Argentina: Universidad de Córdoba.

Safikhani, T., et al. (2014). Thermal Impacts of Vertical Greenery Systems. Malaysia: Universiti Teknologi Malaysia.
Secretaría del Medio Ambiente y Desarrollo Territorial. (2013). Informe Unidad Móvil: El Grullo, Jalisco. Guadalajara: Gobierno del Estado de Jalisco.

Sorensen, M., et al. (1997). Manejo de áreas verdes urbanas. Washintong, D.C.: Inter-American Development Bank, Sustainable Development Department, Environment Division.

Starr, F., et al. (2003). Ficus pumila. Maui, Hawai: United States Geological Survey.

Vélez Restrepo, L. A., y Herrera Villa, M. (2015). Jardines Ornamentales Urbanos Contemporáneos: Transnacionalización, Paisajismo y Biodiversidad. Un estudio exploratoria en Medellin, Colombia. Medellín, Colombia : Revisa Facultad Nacional de Agronomía. 